V. Atomgewichte der Elemente. Von

\title{
H. Bayerlein.
}

Ueber die Festsetzung der Atomgewichte der Elemente für praktisch-analytische Berechnungen. Von Seite einer im Kaiserlichen Gesundheitsamte tagenden Commission analytischer Chemiker ist dem Vorstande der deutschen chemischen Gesellschaft die Frage vorgelegt worden, welche Atomgewichtswerthe den praktisch-analytischen Berechnungen zu Grunde zu legen seien.

Die zur Entscheidung dieser Frage von der genannten Gesellschaft eingesetzte Commission, bestehend aus den Professoren H. Landolt, W. Ostwald und K. Seubert, hat einstimmig beschlossen, folgende Vorschläge zu machen: ${ }^{1}$ )

1. Als Grundlage für die Berechnung der Atomgewichte soll das Atomgewicht des Sauerstoffs gleich 16,000 angenommen werden, und die Atomgewichte der anderen Elemente sollen auf Grund der unmittelbar oder mittelbar bestimmten Verbindungsverhältnisse zum Sauerstoff berechnet werden.

2. Als Atomgewichte der Elemente werden für den Gebrauch der Praxis folgendezur Zeitwahrscheinlichste Werthe vorgeschlagen:

\begin{tabular}{llc|llc} 
Aluminium & $\mathrm{Al}$ & 27,1 & Chlor & $\mathrm{Cl}$ & 35,45 \\
Antimon & $\mathrm{Sb}$ & 120 & Chrom & $\mathrm{Cr}$ & 52,1 \\
Argon (?) & $\mathrm{A}$ & 40 & Eisen & $\mathrm{Fe}$ & 56,0 \\
Arsen & $\mathrm{As}$ & 75 & Erbium (?) & $\mathrm{Er}$ & 166 \\
Baryum & $\mathrm{Ba}$ & 137,4 & Fluor & $\mathrm{F}$ & 19 \\
Beryllium & $\mathrm{Be}$ & 9,1 & Gallium & $\mathrm{Ga}$ & 70 \\
Blei & $\mathrm{Pb}$ & 206,9 & Germanium & $\mathrm{Ge}$ & 72 \\
Bor & $\mathrm{B}$ & 11 & Gold & $\mathrm{Au}$ & 197,2 \\
Brom & $\mathrm{Br}$ & 79,96 & Helium (?) & $\mathrm{He}$ & 4 \\
Cadmium & $\mathrm{Cd}$ & 112 & Indium & $\mathrm{In}$ & 114 \\
Caesium & $\mathrm{Cs}$ & 133 & Iridium & $\mathrm{Ir}$ & 193.0 \\
Calcium & $\mathrm{Ca}$ & 40 & Jod & $\mathrm{J}$ & 126,85 \\
Cerium & $\mathrm{Ce}$ & 140 & Kalium & $\mathrm{K}$ & 39,15
\end{tabular}

1) Berichte d. deutsch. chem. Gesellschaft zu. Berlin 31, 2761 (1898). 


\begin{tabular}{llc|llc} 
Kobalt & Co & 59 & Scandium & $\mathrm{Sc}$ & 44,1 \\
Kohlenstoff & $\mathrm{C}$ & 12,00 & Schwefel & $\mathrm{S}$ & 32,06 \\
Kupfer & $\mathrm{Ca}$ & 63,6 & Selen & $\mathrm{Se}$ & 79,1 \\
Lanthan & $\mathrm{La}$ & 138 & Silber & $\mathrm{Ag}$ & 107,93 \\
Lithium & $\mathrm{Li}$ & 7,03 & Silicium & $\mathrm{Si}$ & 28,4 \\
Magnesium & $\mathrm{Mg}$ & 24,36 & Stickstoff & $\mathrm{N}$ & 14,04 \\
Mangan & $\mathrm{Nn}$ & 55,0 & Strontium & $\mathrm{Sr}$ & 87,6 \\
Molybdän & $\mathrm{Mo}$ & 96,0 & Tantal & $\mathrm{Ta}$ & 183 \\
Natrium & $\mathrm{Na}$ & 23,05 & Tellur & $\mathrm{Te}$ & 127 \\
Neodym (?) & $\mathrm{Nd}$ & 144 & Thallium & $\mathrm{Tl}$ & 204,1 \\
Nickel & $\mathrm{Ni}$ & $58,7 *$ & Thorium & $\mathrm{Th}$ & 232 \\
Niobium & $\mathrm{Nb}$ & 94 & Titan & $\mathrm{Ti}$ & 48,1 \\
Osmium & $\mathrm{Os}$ & 191 & Uran & $\mathrm{U}$ & 239,5 \\
Palladium & $\mathrm{Pd}$ & 106 & Vanadin & $\mathrm{V}$ & 51,2 \\
Phosphor & $\mathrm{P}$ & 31,0 & Wasserstoff & $\mathrm{H}$ & 1,01 \\
Platin & $\mathrm{Pt}$ & 194,8 & Wismuth & $\mathrm{Bi}$ & $208,5 *$ \\
Praseodym (?) & $\mathrm{Pr}$ & 140 & Wolfram & $\mathrm{W}$ & 184 \\
Quecksilber & $\mathrm{Hg}$ & 200,3 & Ytterbium & $\mathrm{Yb}$ & 173 \\
Rhodium & $\mathrm{Rh}$ & 103,0 & Yttrium & $\mathrm{Y}$ & 89 \\
Rubidium & $\mathrm{Rb}$ & 85,4 & Zink & $\mathrm{Zn}$ & 65,4 \\
Ruthenium & $\mathrm{Ru}$ & 101,7 & Zinn & $\mathrm{Sn}$ & $118,5 *$ \\
Samarium (?) & $\mathrm{Sa}$ & 150 & Zirconium & $\mathrm{Zr}$ & 90,6 \\
Sauerstoff & $\mathrm{O}$ & 16,00 & & &
\end{tabular}

Zu der Tabelle macht die Commission folgende Bemerkungen:

Die Zahlen sind im Allgemeinen nur mit so viel Stellen gegeben, dass noch die letzte als sicher angesehen werden kann. Demgemäss sind die von Stas ermittelten Atomgewichte, bei denen die Fehler 3-6 Einheiten der dritten Stelle betragen, mit zwei Stellen, die besser bestimmten anderen Atomgewichte mit einer Stelle, und die weniger sicher bestimmten ohne Decimalstelle angegeben worden. Von dieser Regel ist nur bei Nickel, Wismuth und Zinn, welche mit* bezeichnet sind, abgegangen worden. Im ersten Falle geschah dies, um die wohl unzweifelhaft nachgewiesene Verschiedenheit zwischen den Atomgewichten des Kobalts und Nickels zum Ausdruck zu bringen, obwohl ein Abweichen von $\pm 0,2$ Einheiten bei den beiden angeführten Werthen nicht ansgeschlossen ist.

Bezüglich Wismuth und Zinn ist ähnliches zu bemerken; die wahren Atomgewichte liegen wahrscheinlich näher an den angegebenen Werthen 
als an den nächst liegenden ganzen Zahlen, während sie doch nicht auf 0,1 sicher sind.

Für Wasserstoff ist der Werth 1,008 als auf 0,001 sicher zu betrachten. Doch ist mit Rücksicht auf die Praxis die Abrundung auf 1,01 zugelassen worden, da hierdurch nur ein Fehler von $1 / 5 \%$ bedingt wird.

Die Elemente, deren Namen in der Tabelle mit ? versehen wurden, sind mit Unsicherheiten entweder hinsichtlich ihrer Homogenität oder bezüglich ganzer Einheiten ihrer Atomgewichtswerthe behaftet.

Hinsichtlich der von der Commission den Berathungen zu Grunde gelegten Gesichtspunkte kann an dieser Stelle nur auf das Original verwiesen werden.

Auch Th. W. Richards. ${ }^{1}$ ) hat die bis jetzt vorliegenden Atomgewichtswerthe kritisch gesichtet und die wahrscheinlichsten Werthe für die einzelnen Elemente in einer Tabelle zusammengestellt.

Da dieselbe von der oben genannten Commission mit benutzt worden ist und nur in ganz wenigen Punkten von den Werthen abweicht, welche in der Tabelle auf S. 138 und 139 abgedruckt sind, so verweise ich hinsichtlich dieser Arbeit auf das Original.

1) American chemical Journal 20, 543.

$$
\text { B e ri c h tigu } \mathrm{ng} \text {. }
$$

Im Jahrgang 37 dieser Zeitschrift Seite 662 lies in der Zusammenstellung der Weissweine des Jahrganges 1895 bei Nr. 20 unter Zucker "0,07" statt „0,97“. 